Articulo

\title{
Condiciones socioeconómicas de los productores de maíz de los municipios de Villaflores y La Trinitaria, Chiapas, México
}

\author{
Salvador González Flores ${ }^{1}$ \\ Lenin G. Guajardo-Hernández ${ }^{1 \S}$ \\ Silvia X. Almeraya-Quintero ${ }^{1}$ \\ Luz María Pérez-Hernández ${ }^{1}$ \\ Dora Ma. Sangerman-Jarquín ${ }^{2}$
}

${ }^{1}$ Posgrado en Socio Economía, Estadística e Informática-Desarrollo Rural-Colegio de Posgraduados. Carretera México-Texcoco km 36.5, Montecillo, Texcoco, Estado de México. CP. 56230. Tel. 595 9520200, ext. 1876. (daligonzalez90@hotmail.com; xalmeraya@colpos.mx; luzmaph@colpos.mx). ${ }^{2}$ Campo Experimental Valle de México-INIFAP. Carretera Los reyes-Texcoco km 13.5. CP. 56250. Coatlinchan, Estado de México. (sangerman.dora@inifap.gob.mx).

${ }^{\S}$ Autor para correspondencia: glenin@ colpos.mx

\section{Resumen}

En México, el sector agrícola cuenta con una estructura compleja en términos económicos y sociales, que se refleja en desequilibrios socioeconómicos en diversas zonas del país. Esto ha dado como resultado que los productores rurales consideren como forma de subsistencia, varios sistemas de producción y diversificación de actividades no agrícolas, con el fin de incrementar el ingreso, contar con mayores oportunidades de desarrollo y opciones de progreso en el mejoramiento de la calidad de vida. El objetivo de esta investigación fue determinar las condiciones socioeconómicas en las que se encuentran los productores de maíz de los municipios de Villaflores y La Trinitaria, Chiapas, a fin de establecer politicas públicas diferenciadas que contribuyan a mejorar el ingreso y la satisfacción de las necesidades básicas de los actores objeto de estudio. Metodológicamente, se aplicó el muestreo estratificado afijación proporcional y el análisis multivariado por conglomerados, mediante los métodos jerárquicos y de agrupamiento Wards. Los resultados determinaron la diferencia entre los grupos de los municipios, que fueron: edad y escolaridad de los productores, ingreso por ventas de producción y actividades no agrícolas, destino del ingreso que perciben, acceso a los servicios de salud, espacios y materiales de las viviendas, y servicios básicos. Se concluye, que es necesario reforzar la política socioeconómica e impulsar iniciativas como parte fundamental de la política de desarrollo rural regional, para que se mantenga equilibrado en los ámbitos social y económico y asegurar un nivel de vida digno para los habitantes de las comunidades rurales.

Palabras clave: calidad de vida, desarrollo rural regional, ingreso, políticas públicas diferenciadas.

Recibido: julio de 2019

Aceptado: septiembre de 2019 


\section{Introducción}

En México, el sector agrícola cuenta con una estructura compleja en términos económicos y sociales, que se refleja en desequilibrios socioeconómicos en diversas zonas del país. Esto ha dado como resultado que los productores rurales consideren como forma de subsistencia, varios sistemas de producción y diversificación de actividades no agrícolas, con el fin de incrementar el ingreso, contar con mayores oportunidades de desarrollo y opciones de progreso en el mejoramiento de la calidad de vida en el medio rural.

En este sentido, existen apoyos que otorga el gobierno federal para el sector rural, mediante programas orientados a la producción agropecuaria y a aspectos sociales, creados para coadyuvar al crecimiento y fortalecer la economía del país. Sin embargo, cabe señalar que, de acuerdo con diversos estudios, los programas no contribuyen al desarrollo económico de las zonas rurales. Según Bazán et al (2011), el modelo neoliberal, crisis económicas y la corresponsabilidad social, así como el hecho de que los programas se convirtieron en mecanismos de manipulación política, prácticas de cacicazgo y corrupción por parte de los encargados en su implementación, no han contribuido en el logro de los programas gubernamentales y han propiciado frenar el desarrollo.

Claro ejemplo se muestra en la creación del Programa Nacional de Solidaridad (Pronasol) en el año de 1988, en un contexto de crisis económica y demanda social creciente rebasada en la capacidad de respuesta de las instituciones (Palacios, 2007). De igual forma, en 1992 nace la Secretaría de Desarrollo Social (Sedesol), cuyo objetivo central era combatir la pobreza entre campesinos, indígenas y colonos de zonas urbano-marginadas.

De acuerdo con Rodríguez y Moctezuma (2008), la realidad en la que viven los productores, no sólo en México, sino a nivel Latinoamérica, ha venido empeorando las condiciones de vida de los pobladores rurales de menores ingresos. En este tenor, las iniciativas implementadas no han garantizado el acceso a los derechos sociales a partir del acceso y mejoramiento de la vivienda, abastecimiento de los servicios básicos, gratuidad de la educación, aumento de la cobertura de salud y empleo, e incremento en el ingreso.

De ahí que, este trabajo de investigación se centró en determinar las condiciones socioeconómicas en las que actualmente se encuentran los productores de maíz de los municipios de Villaflores y La Trinitaria, Chiapas. Lo anterior, como forma de referenciar las peculiaridades esenciales en el aspecto económico y social de los productores agrícolas de la región; a fin de establecer políticas públicas diferenciadas que contribuyan a mejorar el ingreso y satisfacción de las necesidades básicas de los actores objeto de estudio.

\section{Materiales y métodos}

La investigación se enfocó en un análisis cuantitativo, para agrupar a los productores de maíz de los municipios de Villaflores y La Trinitaria, Chiapas. Una vez aglomerados, se analizaron las variables con respecto a las condiciones socioeconómicas en las que actualmente se encuentra la población objetivo. 
En primera instancia, se establecieron las variables tomando en cuenta como guía el Informe de la Evaluación de la Política Social 2018, del Consejo Nacional de Evaluación de la Política de Desarrollo Social (Coneval). De ahí que, las variables a considerar fueron: edad, alfabetismo y escolaridad del productor, ingreso promedio anual por venta de producción y actividades no agrícolas, alimentación, acceso al crédito, servicios de salud, seguridad social, calidad y espacios de vivienda y servicios básicos de la misma.

El muestreo utilizado para el trabajo de investigación fue el estratificado; debido que, en este tipo de muestreo la población se divide en estratos, y tiende a mayor precisión que en comparación al muestreo aleatorio simple. Por lo que de acuerdo con Vivanco (2005), para determinar el tamaño de los estratos del muestreo, se asignaron de acuerdo con el estrato de pertenencia, particularmente, atendiendo el criterio de la concentración de $80 \%$ de productores de maíz en las localidades pertenecientes a los municipios de Villaflores y La Trinitaria, Chiapas.

Posteriormente, con base en el padrón de beneficiarios de Proagro Productivo del ciclo agrícola 2017, se calculó el tamaño de la muestra a partir de la estratificación de la población de cada municipio. En este sentido, para el municipio de Villaflores la estratificación de la población comprendió seis localidades seleccionadas, siendo: Villaflores, Jesús María Garza, Villa Hidalgo, Cuauhtémoc, Domingo Chanona y 16 de septiembre. Y para el municipio de La Trinitaria se determinaron cinco comunidades: La Esperanza, Santa Rita, Trinitaria, Tziscao y El Progreso (Cuadro 1).

Cuadro 1. Estratificación de las localidades del municipio de Villaflores y La Trinitaria, Chiapas.

\begin{tabular}{ccccc}
\hline Municipio & Estrato & Localidad & $\mathrm{Ni}$ & Total \\
\hline Villaflores & 1 & Villaflores & 1 & 844 \\
& 2 & Jesús María Garza & 2 & \\
3 & Villa Hidalgo & 3 & \\
& 4 & Cuauhtémoc & 4 & \\
La Trinitaria & 5 & Domingo Chanona & 5 & \\
& 6 & 16 de septiembre & 6 & \\
& 1 & La Esperanza & 1 & 992 \\
& 2 & Santa Rita & 2 & \\
& 3 & Trinitaria & 3 & \\
& 4 & Tzicao & 4 & \\
\hline
\end{tabular}

Elaborado con datos del Padrón de Beneficiarios de Proagro Productivo (2017).

En cuanto a la cantidad de estratos o localidades a considerar, Kish (1972) establece como referencia un rango de 3 a 10 estratos, dado que se desvirtúa la ventaja que ofrece el agrupamiento de elementos. Respecto a la afijación utilizada para el muestreo estratificado se determinó afijación proporcional, dado que el tamaño de cada estrato en la muestra es proporcional al tamaño del correspondiente estrato de la población. Según Vivanco (2005), esta afijación presenta como ventaja que se genera una muestra autoponderada caracterizada por la misma fracción de muestreo en todos los estratos. 
Posteriormente se aplicó la siguiente fórmula estadística que representa el muestreo estratificado afijación proporcional (Vivanco, 2005):

Tamaño total de la muestra

$$
n=\frac{\Sigma_{i-1}^{l} N_{i} P Q_{i}}{N E+\frac{1}{N} \Sigma_{i-1}^{l} N_{i} P_{i} Q_{i}} \quad E=\frac{d^{2}}{Z_{1-\alpha / 2}{ }^{2}}
$$

Tamaño de cada estrato

$$
n_{i}=n\left(\frac{N_{i}}{\Sigma_{i-1}^{\prime} N_{i}}\right)=n\left(\frac{N_{i}}{N}\right)=n\left(W_{i}\right)
$$

El porcentaje de confianza para esta investigación es $95 \%$ y un error de precisión de 10\%. Dónde: $\mathrm{N}=$ tamaño de la población es de 844 productores para el municipio de Villaflores y 992 productores correspondiente al municipio de La Trinitaria; $\mathrm{N}_{\mathrm{i}}=$ tamaño de la población del estrato; $\mathrm{P}_{\mathrm{i}}=$ proporción esperada de 20\%; $\mathrm{Q}_{\mathrm{i}}=1-\mathrm{P}_{\mathrm{i}}(1-0.2=0.8) ; d=$ error absoluto o precisión de $10 \% ; \mathrm{Z}_{1-\alpha / 2}=$ nivel de confianza de $95 \%$, por tanto $\mathrm{Z}_{1-\alpha / 2}=1.962 ; \mathrm{NE}=$ producto del tamaño de la población por el error de estimación; $\mathrm{n}=$ tamaño total de la muestra; $\mathrm{y} \mathrm{n}_{\mathrm{i}}=$ tamaño de cada estrato.

Entonces, el tamaño de muestra $n$ estimado en el caso del municipio de Villaflores y La Trintiaria, fue de 57 y 58 encuestas, respectivamente; lo que representa el total de la población objetivo de la zona de estudio. En otro tenor, para determinar el tamaño de cada estrato fue mediante la fórmula correspondiente sobre afijación proporcional, obteniendo la muestra para cada estrato tal como se muestra en el Cuadro 2.

Cuadro 2. Tamaño de la muestra por estrato de las localidades de los municipios de Villaflores y La Trinitaria, Chiapas.

\begin{tabular}{ccccc}
\hline Municipio & Estrato & Localidad & Ni & Total \\
\hline Villaflores & 1 & Villaflores & 14 & 57 \\
& 2 & Jesús María Garza & 13 & \\
& 3 & Villa Hidalgo & 12 & \\
& 4 & Cuauhtémoc & 7 \\
La Trinitaria & 5 & Domingo Chanona & 6 \\
& 6 & 16 de septiembre & 5 & \\
& 1 & La Esperanza & 14 & 58 \\
& 2 & Santa Rita & 14 & \\
& 3 & Trinitaria & 14 & \\
& 4 & Tzicao & 9 & \\
\hline
\end{tabular}


Consecutivamente, la selección de los productores a entrevistar fue de manera aleatoria por cada estrato, por lo cual se determinó un intervalo constante de selección. Por otra parte, la encuesta utilizada para la recopilación de información fue de tipo semiestructurada donde se aplicaron de manera uniforme para cada uno de los productores de maíz. Las preguntas de ésta se establecieron cerradas y abiertas. La encuesta se dividió en apartados que contemplaron: a) datos generales del productor; b) aspectos de producción; c) aspectos socioeconómicos; y d) aspectos ambientales.

En cuanto al procesamiento y análisis de la información obtenida, se utilizó el programa IBM SPSS (Statistical Package for the Social Sciences) v25.0, a fin de establecer las condiciones socioeconómicas en la que predominan los productores de maíz en ambos municipios. En este sentido, para clasificar los grupos de productores de cada municipio considerando las variables descritas, se utilizó la técnica estadística multivariada denominada conglomerados o análisis clúster ocupando el método jerárquico. Este análisis permite agrupar elementos en grupos homogéneos al interior y heterogéneos entre sí, en función a las similitudes entre ellos.

Derivado de lo anterior, es importante considerar que cuando existen variables altamente correlacionadas, es posible que tengan mayor peso debido a la formación de los conglomerados (Montanero, 2008). Por otra parte, se requiere tener en cuenta la matriz de correlaciones antes del análisis clúster. En el cual, se obtuvieron los estadísticos descriptivos como mínimos, máximos, media y desviación estándar, para determinar la dispersión promedio de todos los puntos de los datos alrededor de su media grupal (no de la media general).

De esta forma, los elementos principales coadyuvan a formar los conglomerados partir de un conjunto de datos no correlacionados. Asimismo, se ocupó del método de agrupamiento Wards (método de pérdida de la inercia mínima) el cual minimiza la varianza entre cada grupo, el uso de la distancia euclidiana al cuadrado y las 18 variables consideradas con alta correlación significativa entre algunas de ellas. Dado que se busca agrupar variables tratando de lograr la máxima homogeneidad en cada grupo, tamaños similares y la mayor diferencia entre los mismos (Bidogeza et al., 2009). Posteriormente, se agruparon los conglomerados que generan menos aumentos en la suma de las distancias dentro de cada conglomerado. Y se determinó la obtención de tres clusters.

\section{Resultados y discusión}

A partir de la información que se obtuvo de las variables principales, el análisis de conglomerados coadyuvó a generar tres grupos de productores, con relación a las condiciones socioeconómicas para cada uno de los municipios del estudio. De esta manera, se presentan en el Cuadro 3 las características socioeconómicas correspondientes al municipio de Villaflores, Chiapas.

Cuadro 3. Características socioeconómicas de productores de maíz del municipio de Villaflores, Chiapas.

\begin{tabular}{lccc}
\hline Nombre de la variable & Grupo I & Grupo II & Grupo III \\
\hline $\begin{array}{l}\text { Porcentaje sobre n (tamaño } \\
\text { de la población muestra) }\end{array}$ & $68 \%$ & $23 \%$ & $9 \%$ \\
$\begin{array}{l}\text { Edad del productor (años) } \\
\text { Sabe leer y escribir }\end{array}$ & 61 a 70 & 31 a 60 & Más de 70 \\
\hline
\end{tabular}


Rev. Mex. Cienc. Agríc. vol. 10 núm. 728 de septiembre - 11 de noviembre, 2019

\begin{tabular}{|c|c|c|c|}
\hline Nombre de la variable & Grupo I & Grupo II & Grupo III \\
\hline $\begin{array}{l}\text { Escolaridad del } \\
\text { productor(años) }\end{array}$ & Primaria & Secundaria & Primaria \\
\hline $\begin{array}{l}\text { Ingreso promedio anual por } \\
\text { venta de producción }(\$)\end{array}$ & $\begin{array}{l}\text { De } \$ 10001 \mathrm{a} \\
\quad \$ 15000\end{array}$ & $\begin{array}{l}\text { De } \$ 10001 \mathrm{a} \\
\quad \$ 15000\end{array}$ & $\begin{array}{c}\text { De } \$ 5001 \mathrm{a} \\
\quad \$ 10000\end{array}$ \\
\hline $\begin{array}{l}\text { Ingreso promedio anual por } \\
\text { actividad no agrícola }(\$)\end{array}$ & $\begin{array}{l}\text { De } \$ 5001 \mathrm{a} \\
\quad \$ 10000\end{array}$ & $\begin{array}{l}\text { De } \$ 35001 \text { a } \\
\quad \$ 45000\end{array}$ & De $\$ 1000$ a $\$ 5000$ \\
\hline $\begin{array}{l}\text { Principal destino del } \\
\text { ingreso que percibe de lo } \\
\text { agrícola y no agrícola }\end{array}$ & $\begin{array}{l}\text { Alimentos, } \\
\text { servicios básicos de } \\
\text { la vivienda y } \\
\text { servicios médicos }\end{array}$ & $\begin{array}{c}\text { Alimentos, } \\
\text { servicios básicos de } \\
\text { la vivienda y } \\
\text { servicios médicos }\end{array}$ & Alimentos \\
\hline $\begin{array}{l}\text { Acceso a los servicios de } \\
\text { salud (atención médica) }\end{array}$ & $\begin{array}{l}\text { Centro de salud u } \\
\text { hospital público }\end{array}$ & $\begin{array}{l}\text { Clínica u hospital } \\
\text { privado }\end{array}$ & $\begin{array}{l}\text { Consultorios del Dr. } \\
\text { Simi }\end{array}$ \\
\hline $\begin{array}{l}\text { Muros de vivienda } \\
\text { (material) }\end{array}$ & $\begin{array}{l}\text { Tabique, Ladrillo o } \\
\text { Block }\end{array}$ & $\begin{array}{l}\text { Tabique, Ladrillo o } \\
\text { Block }\end{array}$ & Adobe \\
\hline $\begin{array}{l}\text { Techo de vivienda } \\
\text { (material) }\end{array}$ & Madera & Teja & Lamina \\
\hline Piso de vivienda (material) & $\begin{array}{l}\text { Cemento o piso } \\
\text { firme }\end{array}$ & $\begin{array}{l}\text { Cemento o piso } \\
\text { firme }\end{array}$ & $\begin{array}{l}\text { Tierra y de cemento } \\
\text { o piso firme }\end{array}$ \\
\hline $\begin{array}{l}\text { Espacios de vivienda } \\
\text { (cantidad) }\end{array}$ & 4 & 5 & 3 \\
\hline Energía eléctrica & Sí & Sí & Sí \\
\hline Drenaje & Sí & Sí & Sí \\
\hline Agua potable & Sí & Sí & Sí \\
\hline Recolección de basura & Sí & Sí & Sí \\
\hline
\end{tabular}

Elaborado con datos de campo (2018).

Grupo I: productores de maíz con edad que oscila entre los 61 y 70 años, saben leer y escribir con nivel académico primaria. Comprende 68\% de la población (39 agricultores de la muestra), con ingresos relativos de $\$ 10001$ a $\$ 15000$ pesos por la venta de producción, sin acceso al crédito, tipo de vivienda independiente y propia, y con acceso a los servicios básicos de la misma.

Grupo II: productores con edad que ronda entre los 31 y 60 años, saben leer y escribir con nivel escolar secundaria. Comprende 23\% de la población (13 agricultores de la muestra), con ingresos relativos de $\$ 10001$ a $\$ 15000$ pesos por la venta de producción y percepción económica adicional por actividades no agrícolas que data entre \$35001 a \$45000 pesos, sin acceso al crédito, tipo de vivienda independiente y propia y con acceso a los servicios básicos de la misma.

Grupo III: productores de maíz con más de 70 años, saben leer y escribir con nivel escolar primaria. Comprende $9 \%$ de la población (5 agricultores de la muestra), con ingresos menores de los \$10 000 pesos por la venta de producción, sin acceso al crédito, tipo de vivienda independiente y propia, y con acceso a todos los servicios básicos de la casa. El Cuadro 4 muestra las características socioeconómicas de los productores del municipio de La Trinitaria, Chiapas. 
Cuadro 4. Características socioeconómicas de productores de maíz del municipio de La Trinitaria, Chiapas.

\begin{tabular}{|c|c|c|c|}
\hline Nombre de la variable & Grupo I & Grupo II & Grupo III \\
\hline $\begin{array}{l}\text { Porcentaje sobre } n \text { (tamaño } \\
\text { de la población muestra) }\end{array}$ & $31 \%$ & $48 \%$ & $21 \%$ \\
\hline Edad del productor (años) & 41 a 50 & 51 a 70 & Más de 70 \\
\hline Sabe leer y escribir & Sí & Sí & Sí \\
\hline $\begin{array}{l}\text { Escolaridad del } \\
\text { productor(años) }\end{array}$ & Primaria & Primaria & Primaria \\
\hline $\begin{array}{l}\text { Ingreso promedio anual por } \\
\text { venta de producción }(\$)\end{array}$ & $\begin{array}{l}\text { De } \$ 5001 \mathrm{a} \\
\quad \$ 10000\end{array}$ & $\begin{array}{l}\text { De } \$ 5001 \mathrm{a} \\
\quad \$ 10000\end{array}$ & $\begin{array}{c}\text { De } \$ 5001 \mathrm{a} \\
\$ 10000\end{array}$ \\
\hline $\begin{array}{l}\text { Ingreso promedio anual por } \\
\text { actividad no agrícola }(\$)\end{array}$ & $\begin{array}{l}\text { De } \$ 1000 \mathrm{a} \\
\quad \$ 5000\end{array}$ & $\begin{array}{c}\text { De } \$ 25001 \\
\$ 35000\end{array}$ & Cero pesos \\
\hline $\begin{array}{l}\text { Principal destino del ingreso } \\
\text { que percibe por ingreso } \\
\text { agrícola y no agrícola }\end{array}$ & $\begin{array}{c}\text { Alimentos, } \\
\text { servicios básicos } \\
\text { de la vivienda y } \\
\text { servicios médicos }\end{array}$ & $\begin{array}{c}\text { Alimentos, servicios } \\
\text { básicos de la } \\
\text { vivienda y servicios } \\
\text { médicos }\end{array}$ & Alimentos \\
\hline $\begin{array}{l}\text { Acceso a los servicios de } \\
\text { salud (atención médica) }\end{array}$ & No se atiende & $\begin{array}{l}\text { Centro de salud u } \\
\text { hospital público }\end{array}$ & $\begin{array}{l}\text { Centro de salud u } \\
\text { hospital público }\end{array}$ \\
\hline $\begin{array}{l}\text { Muros de vivienda } \\
\text { (material) }\end{array}$ & Madera & Madera & Adobe \\
\hline $\begin{array}{l}\text { Techo de vivienda } \\
\text { (material) }\end{array}$ & Lamina & Lamina & $\begin{array}{l}\text { Paja, Palma o } \\
\text { Lámina }\end{array}$ \\
\hline Piso de vivienda (material) & $\begin{array}{l}\text { Cemento o piso } \\
\text { firme }\end{array}$ & $\begin{array}{l}\text { Cemento o piso } \\
\text { firme }\end{array}$ & $\begin{array}{c}\text { Tierra y de cemento } \\
\text { o piso firme }\end{array}$ \\
\hline $\begin{array}{l}\text { Espacios de vivienda } \\
\text { (cantidad) }\end{array}$ & 2 & 3 & 1 \\
\hline Energía eléctrica & Sí & Sí & Sí \\
\hline Drenaje & No & No & No \\
\hline Agua potable & Sí & Sí & No \\
\hline Recolección de basura & No & Sí & No \\
\hline
\end{tabular}

Elaborado con datos de campo (2018).

Grupo I: la edad de los productores fluctuó entre los 41 a 50 años, saben leer y escribir y cuentan con estudios de primaria. Comprende $31 \%$ de la población (18 agricultores de la muestra), con ingresos relativos de $\$ 5001$ a $\$ 10000$ pesos por la venta de producción, sin acceso al crédito, tipo de vivienda independiente y propia, y con acceso a energía eléctrica y agua potable.

Grupo II: productores con edad que oscila entre los 51 y 70 años, saben leer y escribir, con nivel escolar primaria. Comprende $48 \%$ de la población (28 agricultores de la muestra), con ingresos relativos de \$5001 a \$10 000 pesos por la venta de producción y percepción económica adicional por trabajos no agrícolas que oscila entre \$25001 a \$35000 pesos, sin acceso al crédito, tipo de vivienda independiente y propia y con acceso a la mayoría de los servicios básicos. 
Grupo III: la edad de los productores de maíz con más de 70 años, saben leer y escribir y tiene grado académico de primaria. Comprende $21 \%$ de la población (12 agricultores de la muestra), con ingresos de \$5 001 a \$10 000 pesos por la venta de producción, sin acceso al crédito, tipo de vivienda independiente y propia, y sólo cuentan con energía eléctrica. Con relación en los componentes principales que determinaron la diferencia entre los grupos del municipio están: edad y escolaridad de los productores, el ingreso por ventas de producción y actividades no agrícolas, el destino del ingreso que perciben, acceso a los servicios de salud, espacios y materiales de las viviendas y servicios básicos.

En cuanto a la edad y escolaridad, en primera instancia es de notar que, en el municipio de Villaflores, el grupo II cuenta una edad menor que en comparación a los grupos I y III. Lo anterior, conlleva que este grupo tiene mayor nivel de estudios y puede dedicarse a algunas actividades o trabajos adicionales no agrícolas tales como: los negocios comerciales (tienda, comercio informal, molinos de nixtamal) o prestación de servicios (maquila, carpintería, albañilería, herrería, etc).

Por otra parte, para el municipio de La Trinitaria, se refleja que en los tres grupos difiere la edad, pero cuentan con el mismo nivel escolar de primaria. De ahí que el grupo II es con la de mayor porcentaje de población muestra, y en la que aún sólo teniendo estudios de primaria y una edad que oscila entre los 51 y 70 años, generan ingresos por cría o explotación de ganado. En contraste, el grupo I con menor edad de los tres grupos, sus percepciones económicas son inferiores que los otros grupos, dada su limitado nivel de estudios para dedicarse a algún oficio especializado.

De esta manera, se corrobora con lo que argumenta De Janvry y Sadoulet (2001), en lo que respecta al papel que desempeña la educación y la edad para acceder a mejores posibilidades de trabajo remunerado no agrícola. Además, el acceso a la educación en la región requiere de estrategias donde se contrarreste el rezago educativo, que, en ocasiones, se ha trasmitido en generaciones, lo que impacta el desarrollo de capacidades. Tal y como indica Robeyns (2009), ante la ausencia de oportunidades, la educación coadyuva a afianzar el bienestar de las personas y a mejorar el acceso a las condiciones de vida. Es decir, aumentar las libertades humanas en un proceso que expande las capacidades personales, toda vez que amplía las alternativas disponibles para que la gente viva una vida plena (Fukuda, 2004).

Adicionalmente, Bernal (2014) enfatiza que la educación favorece la autonomía de las personas y brinda la posibilidad de autogestionar sus vidas, mediante la generación de capacidades nuevas y enriquecimiento personal y social, permitiendo establecer nuevas oportunidades de bienestar y calidad de vida. Con relación al factor económico, la principal actividad económica en las regiones de estudio es la agricultura; en el caso del municipio de Villaflores, el grupo I y II generan un ingreso promedio anual por venta de su producción de $\$ 10001$ a $\$ 15000$ pesos y el grupo III, menor a $\$ 10000$ pesos.

Esto se debe a la cantidad de superficie cosechada y al rendimiento promedio por hectárea. Sin embargo, sí se equipará con el caso del municipio de La Trinitaria, las percepciones económicas de los productores rondan en los tres grupos entre \$5001 y \$10 000 pesos anuales. Asimismo, se observó que en el municipio de La Trinitaria cuentan con menores ingresos en comparación con el municipio de Villaflores. Lo anterior, se debe a que los primeros en su mayoría destinan parte de la producción al autoconsumo y por el contrario, los segundos cuentan con excedentes de producción que los destinan para la venta. 
Asimismo, en cuanto a la generación de ingresos por trabajos no agrícolas, en Villaflores los productores que mayor ingreso tienen son los del grupo II, derivado de la incursión en negocios comerciales, o en su defecto, como prestadores de servicios de algún tipo de oficios tales como: herrería, albañilería, carpintería, entre otros.

Mientras que para La Trinitaria, también resulta que los productores del grupo II tienen la posibilidad de incrementar sus recursos económicos por concepto de cría y explotación de ganado. De esta forma, Viveros (2010) señala que los productores tratan de articular de manera consistente entre la agricultura y la ganadería. Comparado con el grupo III, donde no generan ningún tipo de ingreso bajo este rubro, dado las limitaciones en conocimientos operacionales y edad.

Según Reardon et al. (2001), en ausencia de las fuentes no agrícolas de ingreso en los hogares rurales pobres, la magnitud de la condición de carencias y pobreza sería varias veces mayor que en comparación a productores diversificados en la generación de ingresos. En efecto, como lo refiere Arias (2009), la producción agrícola ha dejado de ser suficiente, por eso los productores diversifican sus fuentes de ingresos mediante actividades pluriactivas. Según Carvalho y Moesch (2013), los beneficios de la diversificación de actividades no agrícolas en el medio rural consisten en el aumento de recursos económicos, que incentivan el desarrollo económico y social.

Bajo esta perspectiva, Cetré (2006) menciona que el ingreso monetario es necesario como referencia de bienestar, en el cual permite la adquisición de bienes y servicios básicos que coadyuven a un nivel de vida óptimo. Sí el ingreso de las personas está por debajo de lo que requieren, se encontraría en un umbral de carencias socioeconómicas. Al respecto, Guzmán et al. (2005) indican que las familias rurales se incorporan cada vez más a actividades distintas de la agricultura, porque representan un apoyo importante para compensar los bajos ingresos agrícolas Hernández y Aguilar (2015). Lo anterior, según Urquía (2014), constata que existe en México un panorama heterogéneo de grandes carencias como la alimentación, y requiere de intervenciones localizadas en ciertos grupos de la población y en ciertas regiones.

Además, añade que las unidades económicas rurales cuyos integrantes se encuentran en condiciones de pobreza, dado los niveles de ingresos menores que cuentan, así como el alza de precios en los alimentos; tienen acceso deficiente de los alimentos y una profunda precariedad. Algunos otros investigadores como López (2015), evidencia la alta vulnerabilidad alimentaria que se encuentra expuesta la población de menor capacidad económica y, por ende, repercuten en el acceso de la alimentación y la seguridad alimentaria.

Relativo al acceso de los servicios de salud y seguridad social, para los dos municipios hay grandes contrastes. En Villaflores los productores del grupo I, recurren a centros de salud y hospitales públicos, el grupo II a clínicas u hospitales privados, y el grupo III a consultorios del Dr. Simi. No obstante, para La Trinitaria, tanto el grupo de productores II y III, se dirigen a centros de salud y hospitales públicos y el grupo I, prefiere no atenderse. Además, este último grupo tiene una edad menor de 50 años y consideran que una enfermedad simple se cura por sí sola.

En este contexto, los productores con mayores posibilidades económicas optan por atenderse en servicios privados; mientras que productores con menor ingreso, recurren a orientación médica en sectores públicos o farmacias que ofrecen servicio a bajo costo; o en su defecto, no visitan a ningún tipo de servicio de salud. Hoy en día, las comunidades rurales tienen acceso al programa implementado por el gobierno a municipios pobres denominado seguro popular. 
En este orden de ideas, Whagstaff (2002) expresa que la pobreza y la salud están interrelacionadas, asociadas a considerables costos de atención sanitaria, limitaciones y oportunidades que se circunscriben en el factor ingreso, en la poca eficacia de las políticas enfocadas a este campo y en la incapacidad de los servicios de salud de llegar a los pobres. Derivado lo anterior, según Pick et al. (2011), mencionan que la pobreza extrema y la mala alimentación, imposibilitan a las personas de las zonas rurales de contar con oportunidades de desarrollo en alimentación y salud.

Por otro lado, Woolcock y Narayan (2000) anuncian con lo que respecta a factores comunitarios considerar las influencias ambientales geográficas, para llegar al centro de salud resulta difícil por las condiciones intransitables en las comunidades rurales. También, el mantener buenas prácticas sanitarias y la disponibilidad de personal existente en servicios de salud locales pone en desventaja a las personas que habitan en el sector rural. Finalmente, concluye indicando que las desigualdades de la salud y utilización de estos servicios reflejan en gran medida desigualdades con respecto a variables de ingresos, localización y características de los hogares rurales.

Con lo anterior, se infiere que se es necesario establecer o rediseñar iniciativas en el ámbito del sector salud, que coadyuven a combatir la desigualdad en este rubro; invertir en infraestructura y personal que atienda las unidades de salud, para mejorar la salud y asegurar un nivel de vida digno para los habitantes. A propósito de la calidad y espacios de la vivienda en la zona del municipio de Villaflores, en primera instancia se constata que el material de las viviendas en el grupo I y II, es de tabique o block en muros, así como adobe para el grupo III. De igual forma, se replica que para los casos del grupo I y II, los pisos son de cemento o piso firme. Contrario al grupo III, que es de tierra o cemento. En cambio, para los techos cada grupo difiere, siendo de madera, teja y lamina, respectivamente. Mientras tanto, se vislumbra que para el municipio de La Trinitaria, los grupos I y II las viviendas están elaboradas de madera en muros, lamina en techos y cemento en pisos.

En cambio, el grupo III es el más desfavorecido, dado que el material de sus casas es de adobe, palma o lámina, y tierra o cemento, en el mismo orden para los fines que se ocuparon. Asimismo, en el caso de los espacios de las viviendas, los grupos de productores de Villaflores cuentan con mayores habitaciones que oscilan entre 3 y 5, comparado con los agricultores de La Trinitaria, donde tienen de 1 a 3 espacios para dormir y lugar para cocinar.

Referente a los servicios básicos de las viviendas, en Villaflores los tres grupos cuentan con energía eléctrica, drenaje, agua potable y recolección de basura del servicio público. Mientras que en La Trinitaria existen diferencias significativas. El grupo II es el que posee mayores servicios, en comparación con el grupo I que tiene energía eléctrica y agua potable; así como sólo energía eléctrica en el grupo III. Este último, cuenta con fosa séptica o retrete y recurren a recolectar agua de los lagos y lagunas de Montebello. En cuanto a la recolección de basura, el grupo I y III, depositan sus residuos sólidos en contenedores o depósitos de la propia comunidad, dado que no cuentan con servicio público para tal fin.

De esa manera, se coincide con Aguilar et al. (2018), que señalan que la carencia por acceso a servicios básicos afecta en su mayoría a los habitantes de comunidades rurales y una tercera parte de la población no carece de acceso a calidad y espacios de la vivienda. Para concluir, se observa que persisten niveles de carencia en este rubro; por tanto, es indispensable focalizar apoyos a corto y largo plazo para mejorar las viviendas. Además, de invertir en la infraestructura pública que coadyuve a universalizar el derecho de los servicios básicos considerando, las particularidades que necesitan cada grupo de productores de las poblaciones rurales. 


\section{Conclusiones}

El cultivo de la tierra sigue siendo el eje rector de la economía como forma de ingreso a los demás trabajos complementarios a la actividad. De igual forma, el trabajo no agrícola contribuye a complementar el ingreso familiar y a contrarrestar mínimamente las fluctuaciones estacionales del ingreso, pero no es un elemento que supere las carencias socioeconómicas y contribuya al desarrollo de las comunidades rurales.

En este tenor, el ingreso es el componente principal del bienestar y efecto multiplicador en los derechos sociales, por lo que se ocupa de apoyos vinculados en la capacidad productiva de los agricultores y la elevación de la escolaridad. Igualmente, se requiere de políticas públicas diferencias e integrales con la participación de diferentes actores sociales que beneficien el sector por medio de alternativas productivas con potencial, impulsar la productividad y el desarrollo de las capacidades, acceso a la alimentación y vivienda; así como inversión en educación, infraestructura y servicios de salud, a fin de brindar mayores oportunidades al acceso a los derechos sociales y cubrir carencias específicas de los productores.

En definitiva, es necesario reforzar la política socioeconómica e impulsar iniciativas y procesos como parte fundamental de la política de desarrollo rural regional, para que se mantenga equilibrado en los ámbitos social y económico y asegurar un nivel de vida digno para los habitantes de las comunidades rurales.

\section{Literatura citada}

Aguilar, E. A. E.; Caamal, C. I. y Portillo, V. M. 2018. Intensidades de pobreza multidimensional en México a nivel municipal. Rev. Mex. Cienc. Agríc. 1(9):251-258.

Arias, P. 2009. La pluriactividad rural a debate. In: la pluriactividad en el campo latinoamericano. In: Carton, G. H. y Martínez, V. L. (Comps.) 1ª . Edición. FLACSO. Ecuador. 309 p.

Bazán, O. A.; Quintero, S. M. L. y Hernández, E. A. L. 2011. Evolución del concepto de pobreza y el enfoque multidimensional para su estudio. Quivera. 1(13):207-219.

Bernal, A. 2014. La función de la educación para la creación de las capacidades centrales. Rev. Edetania. 46(1):123-140.

Bidogeza, J. C.; Berentsen, P. B. M.; Graaff, J. and Oude, L. A. G. J. M. 2009. A typology of farm households for the Umutura Province in Rwanda. Review. Food Sec. 3(1):321-335.

Carvalho, M. S. e Moesch, M. M. 2013. Turismo como fenômeno social e suas implicações no espaço rural. Rev. Bras. Ecoturismo. 6(2):442-457.

Cetré, M. 2006. Pobreza y distribución del ingreso en América Latina, Colombia y Bogotá. Comercio Exterior. 1(56):33-40.

CONEVAL. 2018. Consejo Nacional de Evaluación de la Política Social. Informe de Evaluación de Política Social 2018. México. 13-27 p.

De Janvry, A. and Sadoulet E. 2001. Income strategies among rural households in Mexico: the role off-farm activities. World Development. 3(29):467-480.

Fukuda, P. S. 2004. Informe sobre el desarrollo humano 2004: la libertad cultural en el mundo diverso de hoy. PNUD. Madrid. 1-12 p. 
Guzmán, G. I.; Santiago, C. M. J.; Leos, J. A.; González, L. G.; Martínez, D. M. A. y Salas, G. J. M. 2005. El papel del empleo rural no agrícola en las comunidades rurales: un estudio de casos en Tepeji del Río, Hidalgo, México. Agric. Soc. Des. 2(2):51-63.

Hernández, E. M. C. y Aguilar, G. G. 2015. Programas de transferencias monetarias condicionadas en México y Chile: distribución y modelo probit de la pobreza. Cabernos Prolam/USP. 14(26):121-132.

Kish, G. B. and Donnenwerth, G. V. 1972. Sex differences in the correlates of stimulus seeking. J. Consulting Clinical Psychol. 38(1):42-49.

López, S. R. 2015. Pobreza y seguridad alimentaria: el caso de México. Rev. Cis. 18(1):24-54.

Montanero, F. 2008. Análisis multivariante. Universidad de Extremadura. Cáceres, España. 286 p.

Palacios, A. 2007. Diferencias, limitaciones y alcances de las estrategias de combate a la pobreza en México. In: la política social en México: tendencias y perspectivas. In: Cordera, R. y Cabrera, C. (Coord.) 1ª . Edición. Facultad de Economía UNAM. México. 401 p.

Pick, S.; García, R. G. y Leenen, I. 2011. Modelo para la promoción de la salud en comunidades rurales a través del desarrollo de agencia personal y empoderamiento intrínseco. Universitas Psychologica. 2(10):327-340.

Reardon, T.; Berdegué, J. y Escobar, G. 2001. Rural nonfarm employment and incomes in Latin America: overview and policy implications. World Development. 3(29):395-409.

Robeyns, I. 2009. Capabilities and theories of justice. In: debating global society. In: Martyinetti, E. (Ed.) 1 a . Edición. Feltrinelli. Milan. 61-89 p.

Rodríguez, A. S. y Moctezuma, P. S. 2008. Bienestar y calidad de vida en una comunidad de la sierra totonaca de Veracruz. Rev. Cienc. Soc. Univ. Iberoam. 5(3):1-18.

Urquía, F. N. 2014. La seguridad alimentaria en México. Salud pública en México. 1(56):92-98.

Vivanco, A. M. 2005. Muestreo estadístico: diseño y aplicaciones. Universitaria, SA, España. 209 p.

Viveros, F. C. M.; Gil, M. A.; López, P. A.; Ramírez, V. B.; Guerrero, R. J. D. y Cruz, L. A. 2010. Patrones de utilización de maíz en unidades de producción familiar del Valle de Puebla, México. Trop. Subtrop. Agroecos. 12(3):471-484.

Wagstaff, A. 2002. Pobreza y desigualdades en el sector salud. Rev. Panam. Salud Púb. 11(5):316- 326.

Woolcock, M. and Narayan, M. 2000. Social capital: implications for development theory, research and policy. World Bank Res. 15(2): 225-249. 